\title{
Oclusão Percutânea de Comunicação Interatrial pelo Sistema Único de Saúde: uma Opção Economicamente Viável
}

\author{
Raul Ivo Rossi Filho', João Luiz Langer Manica' ${ }^{1}$, Cristiano de Oliveira Cardoso
}

\section{RESUMO}

A comunicação interatrial $(\mathrm{ClA})$ é uma das malformações congênitas mais frequentes, representando aproximadamente $5 \%$ a $10 \%$ de todos os defeitos cardíacos congênitos. O tipo mais comum é a CIA ostium secundum (CIA OS), que representa cerca de $75 \%$ dessas malformações. Quando descoberta tardiamente ou na ausência de tratamento, além da alteração no exame clínico, pode existir insuficiência cardíaca, arritmias cardíacas, insuficiência mitral, sobrecarga ventricular direita e hipertensão pulmonar. Em casos avançados com hipertensão pulmonar grave e reversão do shunt interatrial (direita para esquerda) instala-se a cianose, o que indica doença cardiopulmonar grave e mortalidade aumentada. Nas últimas décadas, com o aprimoramento das técnicas e dos dispositivos percutâneos, as possibilidades de tratamento dos defeitos congênitos por via percutânea têm crescido vertiginosamente. Vários estudos já demonstraram os excelentes resultados imediatos e a longo prazo da atriosseptoplastia percutânea. Além de proporcionar maior conforto ao paciente, menores taxas de complicações, menor tempo de recuperação intra e extra-hospitalar e eficácia similar à da atriosseptoplastia cirúrgica, em países desenvolvidos, é comprovadamente a opção mais econômica. Não há dúvidas de que, em futuro próximo, a relação custoefetividade desse procedimento será observada também em países em desenvolvimento como o Brasil, em que o custo da prótese, ainda hoje, é o principal obstáculo para a padronização desse procedimento, e em que os valores pagos pelos procedimentos cirúrgicos continuam muito defasados.

DESCRITORES: Comunicação interatrial. Cardiopatias congênitas. Próteses e implantes.

\footnotetext{
1 Instituto de Cardiologia do Rio Grande do Sul - Fundação Universitária de Cardiologia (IC-FUC) - Porto Alegre, RS, Brasil.

Correspondência: Raul Ivo Rossi Filho. Av. Princesa Isabel, 370 Porto Alegre, RS, Brasil - CEP 90620-000

E-mail: rossi.pesquisa@cardiologia.org.br

Recebido em: 1\%/3/2010 • Aceito em: 12/5/2010
}

\begin{abstract}
Percutaneous Closure of Atrial Septal Defects by the Brazilian Health System: an Economically Feasible Option

Atrial septal defect (ASD) is one of the most frequent congenital malformations, representing $5 \%$ to $10 \%$ of all congenital heart defects. The most common type is the ostium secundum ASD (OS ASD), and represents $75 \%$ of these malformations. When diagnosed late or left untreated, in addition to abnormalities in the clinical examination, there may be heart failure, cardiac arrhythmias, mitral insufficiency, right ventricular overload and pulmonary hypertension. In advanced cases with severe pulmonary hypertension and interatrial shunt reversal (right to left), there is cyanosis, which indicates severe cardiopulmonary disease and increased mortality. In the last decades, with the improvement of percutaneous techniques and devices, the possibility of percutaneous treatment of congenital defects has increased dramatically. Several studies have shown excellent early and long-term results of percutaneous atrial septoplasty. In addition to providing greater comfort for patients, lower complication rates, less in-hospital and extra-hospital recovery time and efficacy similar to that of surgical atrial septoplasty in developed countries, it is definitely the most economic option. There is no doubt that in the near future, the cost-effectiveness ratio of this procedure will also be observed in developing countries such as Brazil, where the cost of the prosthesis is, to this day, the major obstacle for the standardization of this procedure and the amounts paid for surgical procedures are outdated.
\end{abstract}

KEY-WORDS: Heart septal defects, atrial. Heart defects, congenital. Prostheses and implants. 
Rossi Filho RI, et al. Oclusão Percutânea de Comunicação Interatrial pelo Sistema Único de Saúde: uma Opção Economicamente Viável. Rev Bras Cardiol Invasiva. 2010;18(2):212-22.

A comunicação interatrial $(\mathrm{ClA})$ é uma das malformações congênitas mais frequentes, representando aproximadamente $5 \%$ a $10 \%$ de todos os defeitos cardíacos congênitos. ${ }^{1}$ Tem predominância em pacientes do sexo feminino e possui quatro formas de apresentação anatomicamente diferentes entre $\mathrm{si}^{2}$ : CIA tipo ostium secundum (CIA OS), CIA tipo ostium primum (CIA OP), defeito do seio venoso (CIA SV) e defeito do seio coronário (CIA SC). O tipo mais comum é a CIA OS, que representa cerca de 75\% dessas malformações.

A CIA OS é um defeito no septo interatrial na fossa oval, resultante de alteração na formação do septum secundum. Em geral é um defeito com posição central e orifício único; entretanto, pode ocorrer mais de um defeito, causando os chamados defeitos múltiplos ou CIA multifenestrada. Apesar de frequentemente ocorrer como afecção isolada, as CIAs estão associadas a outras malformações ou fazem parte de cardiopatias congênitas complexas em cerca de $20 \%$ a $40 \%$ dos casos.

Tal alteração permite um desvio (shunt) de sangue oxigenado do átrio esquerdo para o átrio direito, aumentando o volume de sangue nas cavidades direitas e, consequentemente, o fluxo de sangue na artéria pulmonar (hiperfluxo pulmonar). Se mantido por muitos anos, o hiperfluxo pulmonar pode acarretar sobrecarga nas câmaras cardíacas direitas, aumento da pressão na artéria pulmonar e doença na microcirculação, causando dano pulmonar irreversível.

O diagnóstico da CIA OS na infância geralmente é feito pela presença de sopro durante consulta médica de rotina. As crianças são, em sua maioria, assintomáticas e as manifestações clínicas dos adultos são inexpressivas até a terceira ou quarta décadas de vida. Os pacientes podem apresentar intolerância ao exercício, dor torácica, palpitações, cansaço e sintomas nãocaracterísticos. Quando descoberta tardiamente ou na ausência de tratamento, além da alteração no exame clínico, pode existir insuficiência cardíaca, arritmias cardíacas, insuficiência mitral, sobrecarga ventricular direita e hipertensão pulmonar. Em casos avançados com hipertensão pulmonar grave e reversão do shunt interatrial (direita para esquerda) instala-se a cianose, o que indica doença cardiopulmonar grave. Esse achado é chamado de síndrome de Eisenmenger (inversão do shunt decorrente de hipertensão pulmonar) e resulta em mortalidade elevada.

Vários autores demonstraram, em séries cirúrgicas, benefício na oclusão do defeito em relação ao tratamento clínico. Konstantinides et al. ${ }^{3}$, em estudo retrospectivo, encontraram melhora da sobrevida em 8,9 anos de seguimento de pacientes operados após os 40 anos (95\% no grupo cirúrgico vs. $84 \%$ no grupo tratamento clínico; $\mathrm{P}<0,02)$. Além da redução da mortalidade, houve menor incidência de arritmias atriais e melhora da capacidade funcional dos pacientes submetidos a atriosseptoplastia.

Ratificando esses achados, Murphy et al. ${ }^{4}$, Groundstroem et al. ${ }^{5}$ e Roos-Hesselink et al. ${ }^{6}$ também verificaram melhora da sobrevida, melhora da capacidade funcional e menor número de complicações tardias em pacientes operados com idade inferior a 25 anos. Esses achados sugerem que a CIA deve ser fechada o mais cedo possível, já que o retardo no tratamento está intimamente relacionado a pior prognóstico. Embora os achados desses autores favoreçam o tratamento cirúrgico, os estudos realizados até então eram longitudinais não-randomizados, com limitado poder para demonstrar a superioridade de um tratamento em relação ao outro. Em 2001, Attie et al. ${ }^{7}$ publicaram um estudo randomizado, não-cego, com 473 pacientes com mais de 40 anos de idade, portadores de CIA OS ou CIA SV, submetidos a tratamento clínico ou cirúrgico. Com seguimento médio de 7,3 anos, observou-se redução significativa do aparecimento de insuficiência cardíaca e pneumonia recorrente no grupo submetido a atriosseptoplastia.

Ainda não está bem definida qual a melhor idade para o procedimento. Atualmente, o consenso é que crianças com diagnóstico precoce devam ser tratadas por volta dos 5 ou 6 anos de idade, ou seja, antes do período escolar. Mesmo com diagnóstico ao nascimento, espera-se até essa idade, pois a doença tem evolução lenta e os riscos cirúrgicos são menores em razão do maior peso das crianças. A partir dessa idade, o tratamento deve ser feito o quanto antes, de maneira eletiva.

Nas últimas décadas, com o aprimoramento das técnicas e dos dispositivos percutâneos, as possibilidades de tratamento dos defeitos congênitos por via percutânea têm crescido vertiginosamente. Em diversas cardiopatias congênitas, o tratamento percutâneo já é considerado a terapêutica de escolha, superando os resultados obtidos com a terapêutica cirúrgica.

\section{TRATAMENTO PERCUTÂNEO DAS COMUNICAÇÕES INTERATRIAIS}

A primeira tentativa de fechamento percutâneo de CIA OS foi descrita em 1975 por King et al. ${ }^{8}$ em uma adolescente de 17 anos de idade. Desde então vários dispositivos foram criados, embora com taxas de sucesso limitadas, resultados tardios inadequados, e elevado risco de perfuração e de fratura tardia das hastes. Além disso, uma vez solto o dispositivo, sua recaptura era muito difícil. Em 1997, Sharafuddin et al. ${ }^{9}$ apresentaram os resultados iniciais de uma prótese com componente único, autocentrável e autoexpansível, com a qual se obteve grande melhora das taxas de sucesso, redução das complicações e melhores resultados a longo prazo. O mais importante é que esse dispositivo, por suas características de construção, permitiu ampliar a utilização da técnica para a maioria dos porta- 
Rossi Filho RI, et al. Oclusão Percutânea de Comunicação Interatrial pelo Sistema Único de Saúde: uma Opção Economicamente Viável. Rev Bras Cardiol Invasiva. 2010;18(2):212-22.

dores da doença. Atualmente, esse dispositivo, denominado Amplatzer ${ }^{\circledR}$ (AGA Medical Corporation, Estados Unidos), e a prótese Helex ${ }^{\circledR}$ (W. L. Gore \& Associates, Inc., Estados Unidos) são os únicos liberados para uso clínico, nos Estados Unidos, pelo Food and Drug Administration (FDA) ${ }^{10}$; porém, no Brasil, a Agência Nacional de Vigilância Sanitária (Anvisa) concedeu autorização para uso clínico das próteses Amplatzer ${ }^{\circledR}$, Helex $^{\circledR}$, CardioSeal ${ }^{\circledast}\left(\right.$ NMT Medical Inc., Estados Unidos), Figulla ${ }^{\circledR}$ (Occlutech $\mathrm{GmbH}$, Alemanha), Atriasept ${ }^{\circledR}$ (Cardia Inc., Estados Unidos) e Solysafe ${ }^{\circledR}$ (Swissimplant AG, Suíça). ${ }^{11}$

Desde 1997, quando Sharafuddin et al. ${ }^{9}$ publicaram o primeiro estudo com a prótese de Amplatzer ${ }^{\circledR}$ em porcos, vários autores demonstraram excelentes resultados imediatos com esse dispositivo. Fischer et al. ${ }^{12}$ encontraram taxa de sucesso imediato de 94\%, sem mortalidade e com resultados mantidos a longo prazo. Em registro do Reino Unido ${ }^{13}$ com 100 pacientes submetidos a fechamento de CIA e forame oval por via percutânea, o defeito foi adequadamente fechado em $93 \%$ dos casos. A taxa de complicações foi inferior a $3 \%$ e nenhum óbito foi observado. De acordo com Losay et al. ${ }^{14}$, em uma série de 42 casos de fechamento percutâneo de ClAs amplas com média de $23 \mathrm{~mm}$ de diâmetro distendido, 93\% dos pacientes apresentaram ausência de shunt residual após seis meses do procedimento, com apenas um caso de embolização da prótese para artéria pulmonar adequadamente removida cirurgicamente. Outros autores também demonstraram resultados semelhantes, expressos na Tabela 1.

Atualmente, o seguimento mais longo é de cinco anos, conforme demonstrado por Yew e Wilson. ${ }^{20}$ Nesse estudo, todas as próteses mantiveram-se adequadamente posicionadas e com fechamento apropriado do defeito. Observou-se também regressão da sobrecarga ven- tricular direita e mínima manifestação de insuficiência mitral. Complicações tardias não foram relacionadas ao dispositivo.

No Brasil, dois estudos demonstraram a efetividade da prótese de Amplatzer ${ }^{\circledR} \mathrm{Com}$ resultados superponíveis aos descritos na literatura. Braga et al. ${ }^{18}$ apresentaram a experiência de 49 pacientes submetidos a fechamento percutâneo de ClA com sucesso técnico imediato de $97,8 \%$. Não ocorreram complicações maiores ou casos de morte. Com seguimento médio de 12 meses, observou-se boa evolução e manutenção dos resultados a longo prazo. Em 2007, Cardoso et al. ${ }^{21}$ apresentaram os resultados de 101 pacientes submetidos a oclusão percutânea de CIA no Instituto de Cardiologia do RS/Fundação Universitária de Cardiologia. O tamanho médio do defeito foi de 21,5 mm de diâmetro e o tamanho médio das próteses utilizadas foi de 23,9 mm de diâmetro. Nesse estudo, observaram-se taxas de sucesso de 93\%. Não ocorreram complicações imediatas relacionadas à liberação da prótese. Apenas complicações leves foram descritas, como 1 caso de arritmia com necessidade de tratamento, 3 casos de hematoma no local da punção e 3 casos de cefaleia. Após seguimento médio de 12,8 meses, todas as próteses estavam bem ancoradas, sem shunt residual, exceto por 2 casos com CIA residual.

Complicações imediatas, como perfuração cardía$\mathrm{ca}^{22}$, bloqueio atrioventricular transitório ${ }^{23}$, embolização do dispositivo ${ }^{24,25}$ ou arritmias mais graves, são raras e intimamente relacionadas a curva de aprendizado do operador e seleção adequada do tamanho do dispositivo. Dentre vários autores, as complicações imediatas reportadas são inferiores a $10 \%$, e não são relatados óbitos. As complicações tardias apontadas são endocardite, embolização do dispositivo e erosão de aorta

TABELA 1

Resultados dos principais trabalhos com a prótese de Amplatzer ${ }^{\circledR}$ para fechamento percutâneo da comunicação interatrial tipo ostium secundum

\begin{tabular}{|c|c|c|c|c|c|c|c|}
\hline Autor & Ano & $\mathbf{n}$ & $\begin{array}{c}\text { Sucesso } \\
\text { imediato } \\
(\%)\end{array}$ & $\begin{array}{c}\text { Complicações } \\
\text { imediatas } \\
(\%)\end{array}$ & $\begin{array}{l}\text { Seguimento } \\
\text { (meses) }\end{array}$ & $\begin{array}{c}\text { Sucesso } \\
\text { tardio } \\
(\%)\end{array}$ & $\begin{array}{c}\text { Complicações } \\
\text { tardias } \\
(\%)\end{array}$ \\
\hline Chan et al. ${ }^{13}$ & 1999 & 100 & 93 & 5 & 3 & 98,9 & 0 \\
\hline Fischer et al. ${ }^{15}$ & 1999 & 52 & 83 & 0 & 3 & NR & NR \\
\hline Cao et al. ${ }^{16}$ & 2001 & 70 & 81,4 & 1,4 & 6,25 & 91,4 & 0 \\
\hline Taeed et al. ${ }^{17}$ & 2001 & 18 & 56 & 0 & 12 & 100 & 0 \\
\hline Fischer et al. ${ }^{12}$ & 2002 & 236 & 84,7 & 0,84 & 27,6 & 94 & NR \\
\hline Braga et al. ${ }^{18}$ & 2004 & 49 & 97,8 & 0 & 12 & 97,8 & 4,2 \\
\hline Masura et al. ${ }^{19}$ & 2005 & 151 & 79,4 & 0 & 78 & 91,3 & 0 \\
\hline Yew \& Wilson ${ }^{20}$ & 2005 & 25 & 100 & 0 & 57,6 & 100 & NR \\
\hline Cardoso et al. ${ }^{21}$ & 2007 & 101 & 93 & 7 & 12,8 & 98 & 0 \\
\hline
\end{tabular}

$\mathrm{n}=$ número de pacientes; $\mathrm{NR}=$ não relatadas. 
Rossi Filho RI, et al. Oclusão Percutânea de Comunicação Interatrial pelo Sistema Único de Saúde: uma Opção Economicamente Viável. Rev Bras Cardiol Invasiva. 2010;18(2):212-22.

e átrios; entretanto, são descritas apenas como relatos de caso. Existem casos descritos de formação de trombos relacionados à prótese em outros tipos de dispositivos; entretanto, com a prótese de Amplatzer ${ }^{\circledR}$, a taxa desse evento foi inexistente. ${ }^{26,27}$ Nesses raros casos, a anticoagulação por período de três a seis meses mostrou ser efetiva para a resolução do problema. Complicações que requerem tratamento cirúrgico são raras, e a maioria pode ser resolvida por via percutânea. Berdat et al. ${ }^{28}$ relataram $8 \%$ de complicações que necessitaram de cirurgia em 124 pacientes submetidos a fechamento percutâneo de CIA e forame oval. Esses procedimentos, porém, foram realizados na década de 1990, no início do desenvolvimento da técnica. Além disso, outras próteses foram utilizadas e corresponderam a maior número de complicações. Atualmente, a incidência de embolização ou mau posicionamento da prótese que requer resgate cirúrgico acontece em 0 a $3 \%$ dos casos. ${ }^{16-21}$

Evidencia-se, então, que o fechamento percutâneo com a prótese de Amplatzer $^{\circledR}$ apresenta alto índice de sucesso, baixo índice de complicações e manutenção de seus resultados a longo prazo. ${ }^{29}$ Além disso, é de fundamental importância ressaltar a universalidade da prótese de Amplatzer ${ }^{\circledast}$, capaz de ocluir CIAs tão grandes quanto $40 \mathrm{~mm}$ de diâmetro com altas taxas de efetividade e resultados tardios demonstrando a segurança do procedimento.

Outras próteses têm demonstrado resultados semeIhantes aos da prótese de Amplatzer ${ }^{\circledR}$; entretanto, apresentam algumas particularidades que devem ser consideradas no momento da escolha da prótese ideal para determinados tipos de defeitos.

A prótese Cardioseal/Starflex ${ }^{\circledR}$ (NMT Medical Inc., Estados Unidos), desenvolvida a partir das primeiras gerações de próteses com umbrella dupla (Clamshell ${ }^{\circledR}$ e, posteriormente, $\left.\operatorname{Cardioseal}^{\circledR}\right)$, com hastes biarticuladas, superfície recoberta por poliéster e, mais recentemente, com mecanismo flexível de autocentralização que permite a oclusão de defeitos com até $20 \mathrm{~mm}$ de diâmetro, tem demonstrado resultados semelhantes aos obtidos com a prótese de Amplatzer ${ }^{\circledR}$. Butera et al. ${ }^{30}$, comparando os resultados de ambas as próteses em 274 pacientes, demonstraram maior número de pacientes com shunt residual imediatamente após a liberação da prótese e após seis meses do procedimento; entretanto, em nenhum dos casos foi demonstrado shunt residual significativo. Além disso, em relação à taxa de complicações, não se demonstrou diferença significativa entre os dois dispositivos, estando apenas descrita maior dificuldade técnica para liberação da prótese em 10 casos submetidos a fechamento com Cardioseal/ Starflex ${ }^{\circledR}$, sendo 3 desses casos adequadamente ocluídos com a prótese de Amplatzer ${ }^{\circledR}$.

Outra prótese utilizada em diversos países e com resultados animadores publicados é o oclusor septal
Helex $^{\circledR}$. Composto por dois discos flexíveis de nitinol em formato helicoidal, recobertos por uma membrana de politetrafluoretileno (PTFE), a prótese está disponível no mercado em cinco diâmetros $(15 \mathrm{~mm}$ a $35 \mathrm{~mm}$ ) e é habitualmente indicada para defeitos de no máximo $22 \mathrm{~mm}$ de diâmetro estirado. Estudo multicêntrico recente relatou a experiência com a prótese $\mathrm{Helex}^{\circledR} \mathrm{em}$ 143 pacientes com diâmetro médio de CIA de 10 mm, comparativamente a pacientes submetidos a tratamento cirúrgico. ${ }^{31} \mathrm{~A}$ taxa de sucesso do tratamento percutâneo foi de $88 \%$ e as principais causas de insucesso foram mau posicionamento da prótese, compressão de estruturas vizinhas e falta de bordos adequados. A taxa de eventos adversos maiores foi discretamente menor nos pacientes submetidos a tratamento percutâneo, entretanto estatisticamente não-significante $(5,9 \%$ vs. 10,9\%). Após 12 meses de seguimento, 98\% dos pacientes do grupo percutâneo apresentaram oclusão completa do defeito. Experiência inicial de dois centros brasileiros com 7 pacientes portadores de CIA OS com média de $11 \mathrm{~mm}$ de diâmetro $(7,5 \mathrm{~mm}-17 \mathrm{~mm})$ e 3 pacientes portadores de forame oval patente demonstrou resultados satisfatórios. ${ }^{32}$ Dentre os 7 casos de $\mathrm{CIA}$, houve $100 \%$ de sucesso. Evidenciaram-se problemas técnicos que necessitaram de recolhimento da prótese e implante de novo dispositivo em 3 casos. Esse fato possivelmente decorreu da maior complexidade da técnica de implante em comparação com as outras próteses, que demanda treinamento especial do operador e curva de aprendizado mais longa. Entretanto, apesar de ser indicada para oclusão de defeitos menores, seu mecanismo não-autocentrável favorece o fechamento de CIAs múltiplas próximas e septos fenestrados. Pode-se concluir, então, que o oclusor septal Helex $^{\circledR}$ é eficaz e seguro no fechamento de defeitos septais atriais pequenos a moderados.

Recentemente, foi publicado o primeiro estudo em humanos de oclusão percutânea de defeitos do septo interatrial com próteses bioabsorvíveis Biostar ${ }^{\circledR}$ (NMT Medical Inc., Estados Unidos). ${ }^{33}$ As próteses são fabricadas a partir de matriz acelular de colágeno intestinal de porcos montado sobre uma prótese Starflex ${ }^{\circledR}$ MP35N de duplo disco. O remodelamento é gradual e ocorre em um período de 24 meses, durante o qual o colágeno é absorvido e substituído por tecido próprio do paciente. Foram implantadas 59 próteses em 58 pacientes, dentre os quais 4 eram portadores de CIAs e 53 eram portadores de forame oval patente. Todas as próteses foram implantadas adequadamente; entretanto, em 2 casos (1 CIA e 1 forame oval patente) as próteses foram retiradas, tendo sido implantadas novas próteses em decorrência do mau posicionamento ao ecocardiograma transesofágico. Após seis meses, 96\% dos pacientes não apresentavam shunt residual. Não foram observadas complicações maiores relacionadas ao procedimento ou à liberação da prótese, demonstrando efetividade e segurança comparáveis aos resultados obtidos com próteses de material sintético. 
Rossi Filho RI, et al. Oclusão Percutânea de Comunicação Interatrial pelo Sistema Único de Saúde: uma Opção Economicamente Viável. Rev Bras Cardiol Invasiva. 2010;18(2):212-22.

O aparecimento de dispositivos capazes de ocluir diversos tipos de defeitos do septo interatrial permite adequada escolha no momento do procedimento, aumenta a capacidade de oclusão dos defeitos e pode ser um fator importante no intuito de viabilizar economicamente o implante desses dispositivos nos países em desenvolvimento.

\section{BASE RACIONAL PARA UTILIZAÇÃO DA TECNOLOGIA}

Vários estudos têm sido realizados com o intuito de comparar os resultados dos fechamentos percutâneo e cirúrgico dos defeitos do septo interatrial. O primeiro estudo multicêntrico comparando os resultados demonstrou altas taxas de sucesso em ambos os procedimentos (95,7\% para o fechamento percutâneo vs. 100\% para o fechamento cirúrgico). ${ }^{34}$ Entretanto, ao se comparar a incidência de complicações relacionadas ao procedimento, demonstrou-se nítida diferença em favor do procedimento percutâneo. Complicações maiores, como tamponamento cardíaco $(1,9 \%)$ e necessidade de nova cirurgia $(1,3 \%)$, entre outras, ocorreram em 5,4\% dos pacientes submetidos a atriosseptoplastia cirúrgica, ao passo que apenas $1,6 \%$ dos pacientes submetidos a procedimento percutâneo tiveram complicações maiores, como embolização da prótese $(0,5 \%)$, arritmia cardíaca $(0,2 \%)$ e embolia cerebral com recuperação completa $(0,2 \%)$. Complicações menores ocorreram em 18,8\% dos pacientes do grupo cirúrgico vs. 7,2\% dos pacientes do grupo percutâneo. Além da maior incidência de complicações, o tempo de permanência hospitalar foi significativamente maior nos pacientes submetidos a procedimento cirúrgico.

Estudo polonês que comparou os resultados entre o fechamento percutâneo e a atriosseptoplastia cirúrgica em crianças e adultos jovens demonstrou a alta efetividade de ambos, apesar da superioridade, em diversos aspectos do procedimento percutâneo, em um centro do Leste Europeu. ${ }^{35}$ Observou-se menor tempo de hospitalização (7,5 dias vs. 2,2 dias) bem como menor taxa de complicações no grupo com tratamento percutâneo. Apenas $6,4 \%$ dos pacientes tratados por via percutânea tiveram complicações (nenhuma grave), comparativamente a $68,2 \%$ dos pacientes do grupo cirúrgico (1 caso de hemiplegia provavelmente decorrente de circulação extracorpórea). Transfusão de sangue foi administrada a $41 \%$ dos pacientes submetidos a atriosseptoplastia cirúrgica e apenas 1 paciente (2\%) do grupo percutâneo necessitou derivado de sangue. Todas essas diferenças foram estatisticamente significantes.

Butera et al. ${ }^{36}$, comparando os tipos de tratamento em crianças de até 6 anos de idade, demonstraram resultados semelhantes, também com altas taxas de efetividade em ambos os grupos. Observou-se menor tempo de hospitalização no grupo percutâneo (1,2 dia vs. 4,8 dias) assim como menor taxa de complicações comparativamente ao grupo cirúrgico. Um terço dos pacientes do grupo cirúrgico apresentou algum tipo de complicação, sendo um terço dessas complicações classificado como maior (hemiplegia, insuficiência cardíaca, tamponamento cardíaco e derrame pleural com comprometimento hemodinâmico). No grupo submetido a atriosseptoplastia percutânea, 9\% dos pacientes apresentaram algum tipo de complicação e apenas 1 (1\%) paciente apresentou complicação maior (embolização da prótese com necessidade de remoção cirúrgica).

Experiência nacional em crianças com média de 6 anos de idade $^{37}$ comparou as complicações intrahospitalares entre os dois tipos de procedimento e demonstrou resultados muito similares aos dados da literatura. O tempo de internação foi em média de 2,2 dias para o grupo percutâneo e de 7,4 dias para o grupo cirúrgico. Em relação às complicações nos pacientes submetidos a atriosseptoplastia cirúrgica, 3\% dos casos evoluíram com bloqueio atrioventricular total com necessidade de marca-passo, 4\% com derrame pericárdico, $1 \%$ com pneumotórax e $41 \%$ dos pacientes necessitaram de transfusão sanguínea. Nenhuma dessas complicações foi observada no grupo submetido a tratamento percutâneo, bem como não houve complicações relacionadas à liberação da prótese.

Estão bem documentados os efeitos deletérios da circulação extracorpórea na contratilidade miocárdica em pacientes submetidos a cirurgia cardíaca. ${ }^{38}$ Entretanto, não haviam sido documentados os efeitos da circulação extracorpórea em pacientes submetidos a atriosseptoplastia cirúrgica comparativamente a pacientes submetidos a procedimento percutâneo. Estudos prévios documentaram que a função ventricular direita está além do normal nos pacientes portadores de CIA antes da intervenção cirúrgica ou percutânea, em decorrência da sobrecarga volumétrica do ventrículo direito. ${ }^{39-41}$ Mesmo assim, diferentes parâmetros ecocardiográficos demonstraram disfunção ventricular direita sistólica e diastólica imediatamente após a intervenção cirúrgica com permanência dessa disfunção após seis meses do procedimento cirúrgico, o que não ocorre nos pacientes submetidos a fechamento percutâneo. ${ }^{39,41,42}$ Além disso, observa-se também disfunção diastólica persistente do ventrículo esquerdo nos pacientes operados. ${ }^{43}$

Além da disfunção ventricular direita, o remodelamento do ventrículo direito parece ter comportamento diferente após oclusão cirúrgica quando comparado com a oclusão percutânea do defeito. Está bem demonstrado que o remodelamento precoce ocorre nos primeiros dias após o fechamento percutâneo do defeito, com quedas drásticas no tamanho do ventrículo direito mensuradas através do modo-M ou estimativa do volume ventricular, tanto em crianças como em adultos. ${ }^{44-47}$ Essas alterações atingem valores dentro da normalidade na maioria dos pacientes em até um mês após o procedimento percutâneo. ${ }^{48}$ Entretanto, séries 
Rossi Filho RI, et al. Oclusão Percutânea de Comunicação Interatrial pelo Sistema Único de Saúde: uma Opção Economicamente Viável. Rev Bras Cardiol Invasiva. 2010;18(2):212-22.

de pacientes submetidos a reparo cirúrgico são controversas quanto a esse processo, mesmo após alguns anos do procedimento. Isso ocorre possivelmente pelos efeitos deletérios da circulação extracorpórea e do clampeamento aórtico, como isquemia miocárdica temporária, e da pericardiotomia, levando a aderências pericárdicas. ${ }^{5,44,49-52}$

Não há dúvidas sobre a segurança e a efetividade de ambos os tipos de atriosseptoplastia, com excelentes resultados a curto e longo prazos em centros de referência do mundo inteiro, tanto em crianças como em adultos. Além disso, não há registros de mortalidade nas últimas séries descritas na literatura. Entretanto, fica evidente a superioridade do tratamento percutâneo comparativamente ao tratamento cirúrgico em diversos aspectos. É inegável que a necessidade de circulação extracorpórea e de clampeamento aórtico para a realização da atriosseptoplastia cirúrgica é um fator agravante, comprovadamente associado a maior morbidade pela reação inflamatória sistêmica e pelo dano miocárdico provocado. O maior tempo de internação hospitalar bem como o maior número de complicações, além de desconforto ao paciente, geram custos à instituição, pela menor rotatividade de leitos e maior tempo de permanência em Unidade de Terapia Intensiva (UTI), além de maior exposição do paciente a derivados de sangue. Afora o pós-operatório mais prolongado, a recuperação domiciliar também exige maior tempo nos pacientes submetidos a terapêutica cirúrgica ${ }^{53}$, o que acarreta retardo no retorno à atividade escolar ou profissional e custos indiretos à sociedade. Por fim, a ausência de incisão cirúrgica, além de evitar os riscos de infecção de ferida operatória, não submete o paciente aos danos estéticos de uma cicatriz torácica.

Os poucos casos (cerca de 7\% nas grandes séries) em que a atriosseptoplastia percutânea não é efetiva, em decorrência da falta de bordos ou da compressão de estruturas vizinhas, são encaminhados a cirurgia sem maiores intercorrências. Além disso, atualmente os grandes centros ao redor do mundo possuem larga experiência no fechamento percutâneo com diversos tipos de próteses e taxas de complicações relacionadas à liberação da prótese cada vez menores, como resultado da curva de aprendizado.

\section{CUSTOS DA ATRIOSSEPTOPLASTIA PERCUTÂNEA E CIRÚRGICA}

Mesmo com a comprovada superioridade da atriosseptoplastia percutânea quando comparada ao procedimento cirúrgico em diversos aspectos médicos, como complicações relacionadas ao procedimento, exposição a derivados de sangue, tempo de internação e cicatriz cirúrgica, estudos têm sido realizados também com o objetivo de viabilizar economicamente o procedimento percutâneo em relação ao cirúrgico, inclusive em países subdesenvolvidos.
No primeiro estudo que avaliou os custos da atriosseptoplastia percutânea, comparando-a a dois tipos de atriosseptoplastia cirúrgica disponíveis (esternotomia e minimamente invasiva), realizado na Itália, observou-se maior taxa de complicações e maior tempo de internação hospitalar nos grupos cirúrgicos. ${ }^{54}$ Esse fato foi o principal responsável pelo aumento do custo do procedimento cirúrgico (em média 13 mil euros para a minimamente invasiva e 15 mil euros para a esternotomia). O custo do procedimento percutâneo, incluindo o valor do dispositivo, foi, em média, de 12.250 euros.

Outro estudo, realizado nos Estados Unidos, no Estado da Carolina do Norte, utilizou um sistema de análise de custos diretos e indiretos de procedimentos médicos desenvolvido pela Universidade de Duke. ${ }^{55}$ Nesse estudo, os custos foram divididos em categorias relacionadas diretamente com o fechamento do defeito (cuidados intensivos e intermediários, serviços cardiovasculares, serviços cirúrgicos, suprimentos médicos e cirúrgicos, e transporte) e custos adicionais (medicamentos, cuidados respiratórios, serviços radiológicos, exames laboratoriais e banco de sangue). Não foram incluídos o custo do dispositivo, os honorários médicos nem o tempo, fora do trabalho ou escola, necessário para recuperação do paciente. Tanto os custos diretos como os custos indiretos relacionados ao fechamento foram maiores no grupo cirúrgico, em comparação com o grupo percutâneo. O custo total do procedimento percutâneo foi de US\$7.397,00, ao passo que o custo total do procedimento cirúrgico foi de US\$ 15.234,00. Mesmo se considerarmos os honorários do cirurgião similares aos dos hemodinamicistas, o que frequentemente não é a regra, essa diferença tenderia a ser ainda maior pelo maior tempo de acompanhamento clínico dos pacientes submetidos a procedimento cirúrgico. Além disso, a diferença de pelo menos US\$ $8.000,00$ ultrapassa o valor da prótese comercializada nos Estados Unidos, o que, somado a todas as vantagens previamente relatadas, fundamentou a atriosseptoplastia percutânea como tratamento padrão de referência para o tratamento da CIA OS naquele país.

Em outro estudo realizado nos Estados Unidos, utilizando a prótese de Amplatzer ${ }^{\circledR}$ e comparando os desfechos clínicos e o custo total (incluindo o valor do dispositivo) do procedimento percutâneo com o procedimento cirúrgico, os resultados foram semelhantes. ${ }^{56}$ Além da maior taxa de complicações e do maior tempo de internação hospitalar, o custo médio estimado por caso tratado com a prótese de Amplatzer ${ }^{\circledR}$ foi de US\$ 11.541,00 vs. US\$21.780,00 no grupo cirúrgico. Não foi identificada nenhuma situação em que o custo da atriosseptoplastia percutânea fosse igual ao custo da cirurgia.

Na Austrália, foi realizado estudo prospectivo que comparou, além do desfecho clínico, custos hospitalares e impacto familiar dos fechamentos cirúrgico e percutâneo dos defeitos do septo atrial. ${ }^{57}$ Quando 
Rossi Filho RI, et al. Oclusão Percutânea de Comunicação Interatrial pelo Sistema Único de Saúde: uma Opção Economicamente Viável. Rev Bras Cardiol Invasiva. 2010;18(2):212-22.

avaliados apenas os custos diretamente relacionados ao procedimento, o cateterismo se mostrou mais oneroso que o procedimento cirúrgico. Entretanto, a necessidade de maior tempo de internação, enfermagem, exames subsidiários e medicamentos contribuiu para o aumento dos custos da atriosseptoplastia cirúrgica, ultrapassando o valor gasto para a atriosseptoplastia percutânea (12.969 dólares australianos vs. 11.845 dólares australianos). Esse foi o primeiro estudo prospectivo que comparou os custos dos fechamentos percutâneo e cirúrgico dos defeitos do septo atrial, confirmando a hipótese dos estudos retrospectivos de que o uso de dispositivos para a oclusão de CIA em países desenvolvidos é economicamente viável e vantajoso para o sistema de saúde local. Além disso, o tempo despendido pelos pais para o acompanhamento dos filhos durante a internação hospitalar foi significativamente maior nos pacientes submetidos a tratamento cirúrgico, o que também acarreta gastos indiretos pelo tempo fora do trabalho.

No Reino Unido, foi realizado outro estudo prospectivo que comparou, entre outras coisas, os custos de ambos os procedimentos, levando em conta todos os tipos de gastos necessários, inclusive o valor da prótese $\left(\right.$ Amplatzer ${ }^{\circledR}$ ) e os honorários médicos. ${ }^{53}$ As taxas de complicação e o tempo de internação hospitalar foram semelhantes aos de outros estudos previamente relatados. Em relação aos gastos, o custo total médio foi semelhante em ambos os procedimentos (5.375 libras para o procedimento percutâneo e 5.412 libras para o procedimento cirúrgico). Assim como em outros estudos, a principal diferença ocorreu em relação aos custos dos equipamentos necessários para o procedimento (2.974 libras para o grupo percutâneo vs. 408 libras para o grupo cirúrgico) e aos custos de internação hospitalar (300 libras para o percutâneo vs. 2.410 libras para o cirúrgico).

Em 2006, na Guatemala, foram comparados pela primeira vez os custos de ambos os procedimentos, cirúrgico e percutâneo, para oclusão de CIA OS em um país em desenvolvimento. ${ }^{58}$ Foram incluídos todos os custos relacionados a sala de hemodinâmica e bloco cirúrgico (inclusive o custo de funcionários), medicações e material utilizado durante anestesia e procedimento, tempo de internação em UTI e hospitalização, derivados de sangue, testes diagnósticos, além do custo do dispositivo e do equipamento de circulação extracorpórea. O custo total do procedimento percutâneo foi de US\$ 4.781,00 vs. US\$ 3.329,00 para a realização da cirurgia $(P<0,0001)$, sendo o alto valor da prótese nesse país (US\$2.930,00) o principal responsável por essa diferença.

Nesse mesmo ano, estudo realizado na Geórgia observou as vantagens do procedimento percutâneo comparativamente ao cirúrgico para o fechamento da CIA, como a menor taxa de transfusões sanguíneas, o menor tempo de internação hospitalar, e o menor custo do tratamento, concluindo que todos os pacientes com defeitos do septo atrial compatíveis com tratamento percutâneo devem ser encaminhados ao laboratório de hemodinâmica. ${ }^{59}$

Pode-se concluir que a atriosseptoplastia percutânea, além de proporcionar maior conforto ao paciente, com menores taxas de complicações e menor tempo de recuperação intra e extra-hospitalar, possui excelentes resultados imediatos e a médio prazo, e que, em países desenvolvidos, é uma opção mais barata e tão eficaz quanto o procedimento cirúrgico. Não há dúvidas de que, em futuro próximo, essa custo-efetividade do procedimento será observada também em países em desenvolvimento, em que o custo da prótese, ainda hoje, é o principal obstáculo para a padronização desse procedimento, e em que os valores pagos pelos procedimentos cirúrgicos continuam muito defasados.

\section{PROPOSTA DE INCLUSÃO DE OCLUSORES SEPTAIS NO SISTEMA ÚNICO DE SAÚDE}

Quando se propõe a introdução de novas tecnologias, as quais rotineiramente têm custos financeiros elevados, é fundamental que se leve em conta a relação custo-benefício do uso do novo material. Tal relação, habitualmente, é escrutinada mais pelo lado "custo" que pelo lado "benefício".

Embora o item "custo" seja de fundamental importância para a avaliação do impacto econômico-financeiro da introdução de novas tecnologias nas tabelas dos provedores de saúde, tanto privados como públicos, existem aspectos tão ou mais importantes a serem considerados.

Sabe-se que os valores de remuneração de procedimentos da tabela do Sistema Único de Saúde (SUS) estão muito defasados. Na maioria dos casos, o valor do reembolso é, em muito, superado pelo valor do gasto pelos hospitais para a realização dos procedimentos mais complexos. O recente movimento com o objetivo de melhorar a remuneração de profissionais e de hospitais que atendem pacientes portadores de cardiopatias congênitas, em geral provenientes das camadas mais pobres da população, é, sem dúvida, um ato na direção certa.

Embora seja clara a sobrecarga imposta àqueles que tratam de pacientes com doenças ditas de alto custo, não houve redução significativa dos preços de insumos necessários para tais tratamentos. Essa distorção permite que o reembolso por cirurgias cardíacas seja muito baixo, tornando difícil a comparação com os valores reais necessários para a introdução de novos métodos para o tratamento percutâneo das cardiopatias congênitas.

O tão importante item "custo" tem leituras diversas nas diferentes sociedades. Como já referido anteriormente, nos países mais desenvolvidos há nítida van- 
Rossi Filho RI, et al. Oclusão Percutânea de Comunicação Interatrial pelo Sistema Único de Saúde: uma Opção Economicamente Viável. Rev Bras Cardiol Invasiva. 2010;18(2):212-22.

tagem financeira no uso de dispositivos para fechamento de CIAs. Por outro lado, nos países em desenvolvimento, o custo da prótese inviabiliza programas para tratamento percutâneo de defeitos do septo interatrial. A diferença entre essas duas sociedades, além do poder econômico, reside na maturidade do tecido social, que demanda a incorporação de avanços tecnológicos pelos serviços de saúde e sua disponibilização àqueles que deles necessitem. Nas culturas menos evoluídas, a população nem sequer tem acesso ao conhecimento ou a ela é permitido discutir vantagens e desvantagens dos eventuais avanços tecnológicos da área médica.

Nossa realidade é uma mistura das duas: temos uma sociedade que recebe a proteção de uma legislação avançada, especialmente em relação a crianças e adolescentes, e que cada vez mais tem acesso à informação; e outra parcela de nossa população, infelizmente, que ainda não tem pleno conhecimento de seus direitos, não tendo acesso aos serviços mais básicos de saúde.

A utilização de novas tecnologias, portanto, deve permitir o melhor atendimento possível ao indivíduo sem inviabilizar o financiador público. Essa realidade será possível quando o sistema público de saúde for convencido de que existem vantagens não apenas financeiras para o emprego dessa tecnologia e, ao mesmo tempo, viabilize sua introdução no orçamento do Ministério da Saúde por meio da racionalização de custos negociada com a indústria.

Voltando à vital relação "custo-benefício", cabe elaborar sobre a parte "benefício". Não existe qualquer dúvida na literatura internacional de que as técnicas de oclusão de defeitos cardíacos produzem resultados imediatos e tardios equivalentes aos da cirurgia convencional. A segurança dos procedimentos é alta e reproduzível e as complicações, raras e conhecidas. Quando empregadas por pessoal adequadamente treinado, a chance de desperdício de material é mínima e as complicações hospitalares, desprezíveis.

A avaliação de inúmeras séries demonstra com clareza que a permanência hospitalar necessária para esses procedimentos é de apenas 48 horas, enquanto o tratamento cirúrgico convencional tradicionalmente requer permanência hospitalar por um período de seis a sete dias. Em um país em que o sistema público de saúde apresenta grande defasagem entre o número de leitos disponível e a demanda por internações, a redução da média da permanência hospitalar proporcionada pela técnica percutânea ajudaria a aliviar o problema da superlotação nos hospitais de alta complexidade.

Séries brasileiras confirmam esses dados, sendo também importante ressaltar que a utilização de derivados de sangue é muitíssimo incomum quando se utiliza a técnica percutânea. Entretanto, o emprego de derivados de sangue ocorre em aproximadamente 30\% dos pacientes submetidos a atriosseptoplastia cirúrgica, mesmo em centros terciários de tratamento, aumentando os custos do tratamento e potencialmente criando morbidade futura.

Outras vantagens menores, mas não menos importantes, são a ausência de cicatriz cirúrgica, a abolição da necessidade de uso de circulação extracorpórea e a menor agressão psicológica associada às técnicas menos invasivas.

\section{IMPLICAÇÕES ECONÔMICAS DA ATRIOSSEPTOPLASTIA PERCUTÂNEA PELO SISTEMA ÚNICO DE SAÚDE}

Não há dúvida de que o Ministério da Saúde, utilizando sua importância estratégica, poderá obter redução de custos na compra dos diversos dispositivos, de modo a reduzir a já pequena diferença de custos entre os dois procedimentos. Esse fenômeno já é observado em diversos países desenvolvidos, em que o preço do dispositivo é incomparável ao preço em países em desenvolvimento, o que torna a atriosseptoplastia percutânea, além de mais segura e com menos complicações, uma prática ainda mais econômica que a cirurgia.

É sabido que houve recente significativa majoração na tabela SUS de pagamentos hospitalares referentes a cirurgias cardíacas para cardiopatias congênitas; entretanto, esses valores ainda se encontram defasados. De acordo com dados do Departamento de Informática do Sistema Único de Saúde (DATASUS) ${ }^{60}$, o valor médio pago por uma internação de cirurgia cardíaca pediátrica em dezembro de 2007 foi de R\$ 14.612,10. Mesmo que, após negociação do Ministério da Saúde com os fornecedores, ainda reste custo a maior para o procedimento percutâneo, essa diferença poderia ser coberta pela racionalização do uso dos leitos disponíveis em hospitais terciários. Em uma realidade como a nossa, a alta rotatividade de leitos é indiscutivelmente benéfica e lucrativa, acarretando a diminuição do tempo de espera por procedimentos diagnósticos e terapêuticos, com consequente melhora da qualidade e da agilidade dos serviços prestados pelo SUS. Além disso, torna-se desnecessário avaliar o ganho secundário dos pacientes beneficiados por essa tecnologia, já extensamente explicitado.

Considerando a taxa de natalidade atual no Brasil, de 16/1.000 habitantes por ano, e a população de 183 milhões de habitantes, teremos aproximadamente 3 milhões de nascidos vivos ao final de um ano. Levando em conta que entre $0,6 \%$ e $1 \%$ da população em geral é portadora de cardiopatia congênita, e que a incidência de $\mathrm{CIA}$ é de $6 \%$ a $10 \%$ entre todas as cardiopatias congênitas, teremos ao final de um ano entre mil e 3 mil novos casos de CIA na população em geral; entretanto, uma parte dessas comunicações não tem indicação de fechamento. Em 2008, foram realizadas 
Rossi Filho RI, et al. Oclusão Percutânea de Comunicação Interatrial pelo Sistema Único de Saúde: uma Opção Economicamente Viável. Rev Bras Cardiol Invasiva. 2010;18(2):212-22.

1.842 atriosseptoplastias cirúrgicas ${ }^{60}$ pelo SUS. Considerando que aproximadamente $80 \%$ desses defeitos são passíveis de tratamento percutâneo, seriam realizadas em torno de 1.473 atriosseptoplastias percutâneas nesse ano. Se o valor pago pelo procedimento cirúrgico cardíaco pediátrico, atualmente, é de R \$ 14.612,10, em média, e o valor do procedimento percutâneo acrescido do valor da prótese gira em torno de R\$20 mil, podemos calcular que o valor adicional para implementação dessa tecnologia não seria superior a R\$ 8 miIhões por ano, apenas levando em consideração o aspecto econômico da questão. Entretanto, se levarmos em conta o menor tempo de internação necessário para o procedimento percutâneo (dois dias em média) em relação ao procedimento cirúrgico (sete dias em média), teríamos uma economia de 9.210 diárias (pelo menos metade delas de UTI) em 2008, o que poderia colaborar com a diminuição da extensa lista de espera de diversos procedimentos cirúrgicos.

\section{CONCLUSÃO}

A análise da literatura deixa claro que o fechamento percutâneo de defeitos no septo interatrial é tão seguro e eficaz quanto o fechamento cirúrgico, acarretando menor morbidade e menor permanência hospitalar.

A melhoria dos honorários pagos à cirurgia de congênitos e a eventual redução dos preços dos dispositivos, a ser negociada pelo Ministério da Saúde com os eventuais fornecedores de material, permitirão viabilizar, do ponto de vista econômico, a introdução dessa opção de tratamento aos pacientes cobertos pelo SUS.

\section{CONFLITO DE INTERESSES}

Os autores declararam inexistência de conflito de interesses relacionado a este manuscrito.

\section{REFERÊNCIAS}

1. Braunwald E. Heart disease. $6^{\text {th }}$ ed. Philadelphia: Saunders; 2001.

2. Ho YS, Baker EJ, Rigby ML, Anderson RH. Congenital heart disease, morphologic and clinical correlations. Barcelona: Mosby-Wolfe; 1995

3. Konstantinides S, Geibel A, Olschewski M, Gornandt L, Roskamm H, Spillner G, et al. A comparison of surgical and medical therapy for atrial septal defect in adults. $N$ Engl J Med. 1995;333:469-73.

4. Murphy JG, Gersch BJ, Phil D, McGoon MD, Mair DD. Longterm outcome after surgical repair of isolated atrial septal defect. Follow-up at 27 to 32 years. N Engl J Med. 1990;323: 1645-50.

5. Groundstroem KWE, livainen TE, Talvensaari T, Lahtela JT. Late postoperative follow-up of ostium secundum defect. Eur Heart J. 1999;20(12):904-9.

6. Roos-Hesselink JW, Meijboom FJ, Spitaels SE, van Domburg $\mathrm{R}$, van Rijen EH, Utens EM, et al. Excellent survival and low incidence of arrhythmias, stroke and heart failure long-term after surgical ASD closure at young age. Eur Heart J. 2003; $24: 190-7$.
7. Attie F, Rosas M, Granados N, Zabal C, Buendia A, Calderon J. Surgical treatment for secundum atrial septal defect in patients > 40 years old: a randomized clinical trial. J Am Coll Cardiol. 2001;38:2035-42.

8. King TD, Thompson SL, Steiner C, Mills NL. Secundum atrial septal defect. Nonoperative closure during cardiac catheterization. JAMA. 1976;235:2506-9.

9. Sharafuddin MJA, Gu X, Titus JL, Urness M, Cervera-Ceballos JJ, Amplatz K. Transvenous closure of secundum atrial septal defects. Circulation. 1997;95:2162-8.

10. U. S. Food and Drug Administration (FDA) [homepage on the Internet]. [cited 2009 Feb 17]. Available from: http:// www.fda.gov/cdrh/mda/docs/p000039.pdf.

11. Brasil. Ministério da Saúde. Agência Nacional de Vigilância Sanitária [homepage na Internet]. Brasília; c2003. [citado 2009 fev. 17]. Disponível em: http://www7.anvisa.gov.br/ datavisa/Consulta_Produto_correlato/consulta_correlato.asp.

12. Fischer G, Stieh J, Uebing A, Hoffmann U, Morf G, Kramer $\mathrm{HH}$. Experience with transcatheter closure of secundum atrial septal defects using the Amplatzer septal occluder: a single center study in 236 consecutive patients. Heart. 2003; 89:199-204.

13. Chan KC, Godman MJ, Walsh K, Wilson N, Redington A, Gibbs JL. Transcatheter closure of atrial septal defect and interatrial communications with a new self expanding nitinol double disc device (Amplatzer septal occluder): a multicenter UK experience. Heart. 1999;82:300-6.

14. Losay J, Petit J, Lambert V, Esna G, Berthaux X, Brenot P, et al. Percutaneous closure with Amplatzer device is a safe and efficient alternative to surgery in adults with large atrial septal defects. Am Heart J. 2001;142(3):544-8.

15. Fischer G, Kramer $\mathrm{HH}$, Stieh J, Harding P, Jung O. Transcatheter closure of secundum atrial septal defects with the new self-centering Amplatzer septal occluder. Eur Heart J. 1999;20:541-9.

16. Cao QL, Du ZD, Joseph A, Koenig P, Heitschmidt M, Rhodes $\mathrm{J}$, et al. Immediate and six-month results of the profile of the Amplatzer Septal Occluder as assessed by transesophageal echocardiography. Am J Cardiol. 2001;88:754-9.

17. Taeed R, Shim D, Kimball TR, Michelfelder EC, Salaymeh KJ, Koons LM, et al. One-year follow-up of the Amplatzer device to close atrial septal defect. Am J Cardiol. 2001;87:116-8.

18. Braga LNB, Souza AGMR, Pedra CAC, Esteves SRF, Esteves CA, Fontes VF. Efetividade clínica e segurança do tratamento percutâneo da comunicação interatrial tipo ostium secundum com prótese de Amplatzer ${ }^{\circledR}$. Arq Bras Cardiol. 2004;83:7-13.

19. Masura J, Gavora P, Podnar T. Long-term outcome of transcatheter secundum-type atrial septal defect closure using Amplatzer septal occluders. J Am Coll Cardiol. 2005;45:505-7.

20. Yew G, Wilson NJ. Transcatheter atrial septal defect closure with Amplatzer septal occluder: five years follow-up. Catheter Cardiovasc Interv. 2005;64(2):193-6.

21. Cardoso CO, Rossi Filho RI, Machado PR, François LM, Horowitz ES, Sarmento-Leite R. Effectiveness of the Amplatzer device for transcatheter closure of an ostium secundum atrial septal defect. Arq Bras Cardiol. 2007;88:384-9.

22. Vojacek J, Mates M, Popelová J, Pavel P. Perforation of the right atrium and the ascending aorta following percutaneous transcatheter atrial septal defect closure. Interact Cardiovasc Thorac Surg. 2005;4:157-9.

23. Suda K, Raboisson MJ, Piett E, Dahdah NS, Miró J. Reversible atrioventricular block associated with closure of atrial septal defects using the Amplatzer device. J Am Coll Cardiol. 2004; 43:1677-82. 
Rossi Filho RI, et al. Oclusão Percutânea de Comunicação Interatrial pelo Sistema Único de Saúde: uma Opção Economicamente Viável. Rev Bras Cardiol Invasiva. 2010;18(2):212-22.

24. Tsilimingas N, Reiter B, Kodolitsch Y, Münzel T, Meinertz T, Hofmann T. Surgical revision of an uncommonly dislocated self-expanding Amplatzer septal occluder device. Ann Thorac Surg. 2004;78:686-7.

25. Mellert F, Preusse CJ, Haushofer M, Winkler K, Nill C, Pfeiffer D, et al. Surgical management of complications caused by transcatheter ASD closure. Thorac Cardiovasc Surg. 2001; 49(6):338-42.

26. Krumsdorf U, Ostermayer S, Billinger K, Trepels T, Zadan E, Horvath $\mathrm{K}$, et al. Incidence and clinical course of thrombus formation on atrial septal defect and patients foramen ovale closure device in 1000 consecutive patients. J Am Coll Cardiol. 2004;43:302-9.

27. Anzai H, Child J, Natterson B, Krivokapich J, Fishbein MC, Chan VK, et al. Incidence of thrombus formation on the Cardioseal and the Amplatzer interatrial closure devices. Am J Cardiol. 2004;93:426-31.

28. Berdat PA, Chatterjee T, Pfammatter JP, Windecker S, Meier B, Carrel T. Surgical management of complications after transcatheter closure of an atrial septal defect or patent foramen ovale. J Thorac Cardiovasc Surg. 2000;120:1034-9.

29. Chessa M, Carminati M, Butera G, Bini RM, Drago M, Rosti $\mathrm{L}$, et al. Early and late complications associated with transcatheter of secundum atrial septal defect. J Am Coll Cardiol. 2002; 39:1061-5.

30. Butera G, Carminati M, Chessa M, Delogu A, Drago $M$, Piazza L, et al. CardioSEAL/STARflex versus Amplatzer devices for percutaneous closure of small to moderate (up to $18 \mathrm{~mm}$ ) atrial septal defects. Am Heart J. 2004;148(3):507-10.

31. Jones TK, Latson LA, Zahn E, Fleishman CE, Jacobson J, Vincent R, et al. Results of the U.S. Multicenter Pivotal Study of the HELEX Septal Occluder for Percutaneous Closure of Secundum Atrial Septal Defects. J Am Coll Cardiol. 2007; 49(22):2215-21.

32. Pedra CA, Pedra SR, Esteves CA, Pontes SC, Braga SL, Arrieta SR, et al. Experiência inicial no Brasil com a prótese Helex para oclusão percutânea de defeitos interatriais. Arq Bras Cardiol. 2003;81(5):435-43.

33. Mullen MJ, Hildick-Smith D, De Giovanni JV, Duke C, Hillis WS, Morrison WL, et al. BioSTAR Evaluation STudy (BEST). A prospective, multicenter, phase I clinical trial to evaluate the feasibility, efficacy, and safety of the BioSTAR bioabsorbable septal repair implant for the closure of atrial-level shunts. Circulation. 2006;114:1962-7.

34. Du ZD, Hijazi ZM, Kleinmann C, Silverman NH, Larntz K; Amplatzer Investigators. Comparison between transcatheter and surgical closure of secundum atrial septal defect in children and adults: results of a multicenter nonrandomized trial. J Am Coll Cardiol. 2002;11:1836-44.

35. Bialkowski J, Karwot B, Szkutnik M, Banaszak P, Kusa J, Skalski J. Closure of atrial defect in children. Surgery versus Amplatzer ${ }^{\circledR}$ device implantation. Tex Heart Inst J. 2004;31:220-3.

36. Butera G, Lucente M, Rosti L, Chessa M, Micheletti A, Giamberti A, et al. A comparison between the early and midterm results of surgical as opposed to percutaneous closure of defects in the oval fossa in children aged less than 6 years. Cardiol Young. 2007;17:35-41.

37. Rossi Filho RI, Cardoso C, Machado P, François LMG, Horowitz ESK, Sarmento-Leite R. Estudo comparativo entre complicações hospitalares para tratamento percutâneo da prótese de Amplatzer x cirurgia para comunicação interatrial tipo ostium secundum em crianças menores de 10 anos. Arq Bras Cardiol. 2006;87(1):154.

38. Brookes CL, White PA, Bishop AJ, Oldershaw PJ, Redington AN, Moat NE. Validation of a new intraoperative technique to evaluate load-independent indices of right ventricular perfor- mance in patients undergoing cardiac operations. J Thorac Cardiovasc Surg. 1998;116:468-76.

39. Dhilon R, Josen M, Henein M, Redington A. Transcatheter closure of atrial septal defect preserves right ventricular function. Heart. 2002;87:461-5.

40. Pascotto M, Caso P, Santoro G, Caso I, Cerrato F, Pisacane C, et al. Analysis of right ventricular Doppler tissue imaging and load dependence in patients undergoing percutaneous closure of atrial septal defect. Am J Cardiol. 2004; 94:1202-5.

41. Abd El Rahman MY, Hui W, Timme J, Ewert P, Berger F, Dsebissowa F, et al. Analysis of atrial and ventricular performance by tissue Doppler imaging in patients with atrial septal defects before and after surgical and catheter closure. Echocardiography. 2005;22:579-85.

42. Di Salvo G, Drago M, Pacileo G, Carrozza M, Santoro G, Bigazzi MC, et al. Comparison of strain rate imaging for quantitative evaluation of regional left and right ventricular function after surgical versus percutaneous closure of atrial septal defect. Am J Cardiol. 2005;96:299-302.

43. Pawelec-Wojtalik M, Wojtalik M, Mrowczynski W, Surmacz $\mathrm{R}$, Quereshi SA. Comparison of cardiac function in children after surgical and Amplatzer occluder closure of secundum atrial septal defects. Eur J CardioThorac Surg. 2006;29: 89-92.

44. Thilén U, Persson S. Closure of atrial septal defect in the adult. Cardiac remodeling is an early event. Int J Cardiol. 2006;108:370-5.

45. Rossi Filho RI, Cardoso CO, Machado PR, François LMG, Horowitz ESK, Sarmento-Leite R. Transcatheter closure of atrial septal defect with Amplatzer device in children aged less than 10 years old: immediate and late follow-up. Catheter Cardiovasc Interv. 2008;71(2):231-6.

46. Eyskens B, Ganame J, Claus P, Boshoff D, Gewillig M, Mertens L. Ultrasonic strain rate and strain imaging of the right ventricle in children before and after percutaneous closure of an atrial septal defect. J Am Soc Echocardiogr. 2006;19(8):994-1000.

47. Schussler JM, Anwar A, Phillips SD, Roberts BJ, Vallabhan RC, Grayburn PA. Effect on right ventricular volume of percutaneous Amplatzer closure of atrial septal defect in adults. Am J Cardiol. 2005;95:993-5.

48. Pascotto M, Santoro G, Cerrato F, Caputo S, Bigazzi MC, lacono $\mathrm{C}$, et al. Time-course of cardiac remodeling following transcatheter closure of atrial septal defect. Int J Cardiol. 2006;112(3):348-52.

49. Pearlman AS, Borer JS, Clark CE, Henry WL, Redwood DR, Morrow AG, et al. Abnormal right ventricular size and ventricular septal motion after atrial septal defect closure: etiology and functional significance. Am J Cardiol. 1978;41(2):295-301.

50. Meyer RA, Korfhagen JC, Covitz W, Kaplan S. Long-term follow-up study after closure of secundum atrial septal defect in children: an echocardiographic study. Am J Cardiol. 1982; 50(1):143-8.

51. Ning SB, Fazal H, Cook D, Wood MM, Duncan WJ, Rowe RD. Right ventricular size and ventricular septal motion after repair of atrial septal defect in children. Can J Surg. 1984; 27(4):395-8.

52. Attenhofer Jost CH, Oechslin E, Seifert B, Maly F, Fatio R, Turina J, et al. Remodeling after surgical repair of atrial septal defects within the oval fossa. Cardiol Young. 2002; 12(6):506-12.

53. Thomson JD, Aburawi EH, Watterson KG, Van Doorn C, Gibbs JL. Surgical and transcatheter (Amplatzer) closure of atrial septal defects: a prospective comparison of results and cost. Heart. 2002;87(5):466-9. 
Rossi Filho RI, et al. Oclusão Percutânea de Comunicação Interatrial pelo Sistema Único de Saúde: uma Opção Economicamente Viável. Rev Bras Cardiol Invasiva. 2010;18(2):212-22.

54. Formigari R, Di Donato RM, Mazzera E, Carotti A, Rinelli G, Parisi $F$, et al. Minimally invasive or interventional repair of atrial septal defects in children: experience in 171 cases and comparison with conventional strategies. J Am Coll Cardiol. 2001;37(6):1707-12.

55. Baker SS, O'Laughlin MP, Jollis JG, Harrison JK, Sanders SP, Li JS. Cost implications of closure of atrial septal defect. Catheter Cardiovasc Interv. 2002;55(1):83-7.

56. Kim JJ, Hijazi ZM. Clinical outcomes and costs of Amplatzer transcatheter closure as compared with surgical closure of ostium secundum atrial septal defects. Med Sci Monit. 2002; 8(12):787-91.

57. Hughes ML, Maskel G, Goh T, Wilkinson JL. Prospective comparison of costs and short term health outcomes of surgi- cal versus device closure of atrial septal defect in children. Heart. 2002;88(1):67-70.

58. Vida VL, Barnoya J, O'Connell M, Leon-Wyss J, Larrazabal LA, Castañeda AR. Surgical versus percutaneous occlusion of ostium secundum atrial septal defects: results and cost-effective considerations in a low-income country. J Am Coll Cardiol. 2006;47(2):326-31.

59. Khelashvili V, Gogorishvili I, Metreveli I, Tsintsadze A. Comparison of surgical and by transcatheter methods of closure of atrial septal defect based on the two year experience. Georgian Med News. 2006;132:17-20.

60. Brasil. Ministério da Saúde. DATASUS [homepage na Internet]. Brasília; 2009. [citado 2009 fev. 18]. Disponível em: http:/ /tab-net.datasus.gov.br/cgi/tabcgi.exe?sih/cnv/piuf.def. 